\title{
Minkowski space approach for the Bethe-Salpeter equation
}

\author{
Lauro Tomio $^{1,2, \star}$, Cristian Gutierrez ${ }^{1}$, and Vitor Gigante ${ }^{3}$ \\ ${ }^{1}$ Instituto de Física Teórica, UNESP, 01140-070, São Paulo, SP, Brazil \\ ${ }^{2}$ Instituto Tecnológico de Aeronáutica, DCTA, 12.228-900, São José dos Campos, SP, Brazil \\ ${ }^{3}$ Laboratório de Física Teórica e Computacional - LFTC, Universidade Cruzeiro do Sul, \\ 01506-000, São Paulo, SP, Brazil
}

\begin{abstract}
The bound-state energy spectrum for two-boson system with a fixed exchanged massive boson is investigated by considering the scalar Bethe-Salpeter (BS) equation in Minkowski space within the ladder-approximation framework. The approach is extending to excited states a previous study performed for the ground state. The number of states in the spectrum is restricted by the coupling strength of the two-body interaction, as well as the mass of the exchanged boson. In the present approach, we use the Nakanishi Integral Representation and the formally exact null-plane projection. As usual a comparison between eigenvalues obtained in the Minkowski and Euclidean space is presented. Within such scheme, the light-front momentum-space valence wave functions are obtained for the ground and excited states, together with the corresponding quantities in the impactparameter space. Among relevant features emerging from this study, we can point out the node structure of light-front wave function and the leading exponential fall-off of the valence wave function in the impact-parameter space.
\end{abstract}

\section{Introduction}

The study of the relativistic bound-state problem, by means of the fully covariant Bethe-Salpeter (BS) equation [1] directly in the Minkowski space, has been undertaken in the last two decades thanks to the Nakanishi integral representation (NIR) to represent the BS amplitude [2]. The NIR leads to an integral equation for the Nakanishi weight-function, which is equivalent to the initial BS equation. The first attempts to use the NIR in the non-perturbative regime were performed by K. Kusaka and A.G. Williams [3, 4], when studying a bound-state system composed by two scalar particles interacting by means of another scalar, in the ladder approximation. To treat the singular behavior of the integral equation a suitable numerical treatment was adopted. Within the same problem, a substantial simplification in the derivation of the equation for the Nakanishi weight-function was achieved in the work developed by J. Carbonell and V. Karmanov [5]. In addition of using the NIR for the BS amplitude, the light-front (LF) projection was implemented in a twofold in [5], in order to remove the singularities present in the integral equation of the Nakanishi weight-function and to obtain the LF valence wave function for the bound state. This method is shown to be valid for any irreducible kernel in the BS equation. Further simplifications to the integral equation derived in [5] were achieved by using the uniqueness conjecture of the Nakanishi weight-function, as shown in [6,7] by considering

\footnotetext{
^e-mail: tomio@ift.unesp.br
} 
the ladder kernel. The exploration of the method to include kernels beyond the ladder has successfully been performed in $[8,9]$. Moreover, in [10], within the NIR the effect of the self-energy contribution to the binding energy of the ground state has been explored in the scalar theory. It is important to point out that the very relevant problem of two-fermion bound state has been investigated within the NIR framework in $[11,12]$, for the case $J^{\pi}=0^{+}$, where $J$ is the total spin and $\pi$ the parity of the bound state.

The previous successful applications of the NIR for the BS equation in Minkowski space encouraged us to formulate an extended program to investigate other interesting problems, by considering the NIR together with LF projection methods. As a natural extension of the previous analysis, which was limited only for ground states, within a recent work done in [13], the $s$-wave spectrum of excited states for the scalar theory was explored in the ladder kernel approximation. This problem, as far as we know, was for the first time investigated in the Minkowski space, with relevant details appearing in [14]. In the present communication, we complement the results presented in [14] with some further analysis considering the results obtained in [13]. In addition of computing the binding energies, which are available in the Euclidean space, the method allows one to obtain the LF wave function, which should reveal the node structure typical for excited states. As usual, the calculation in the Euclidean space is used to verify the reliability of the method by comparing the eigenvalues in Minkowski and Euclidean spaces. In order to widen the applicability of the calculations in Minkowski space, we explore the structure of the valence wave function in the impact parameter (IP) space. This quantity is obtained as the Fourier transform of the transverse momentum of the LF wave function, while keeping the longitudinal component. Thus, it provides a correlation between the longitudinal momentum of the constituent with its transversal probability distribution. We were motivated to study this quantity because of the increasing interest in hadron physics, when investigating structural properties of hadrons, such as the parton transverse-momentum distributions [15, 16]. A detailed description of the wave function in the IP space is presented here for the first-excited state.

Next, in Sec. 2 we review the NIR method to study bound states, with particular focus on an extension of the approach to obtain the excited states. In Sec. 3, in order to enhance the confidence on the approach and appreciate its numerical performance, we include a comparison between the eigenvalues obtained in the Minkowski and Euclidean spaces. The consistency of the results are also verified for the eigenvalues in the non-relativistic limit. In Sec. 4, we discuss the results obtained for the LF momentum-space valence wave function, considering the ground and first-excited states. The valence wave function in the impact-parameter space is analysed in Sec. 5, presenting our results corresponding to the first excited state. Finally, in Sec. 6, we resume this report with a brief conclusion.

\section{Minkowski space Bethe-Salpeter equation and bound-state spectrum}

The BS equation for a bound state composed by two scalar particles of equal mass $m$ in the ladder approximation, without considering vertex neither self-energy corrections, reads as $[6,7,14]$ :

$$
\varphi(k, p)=\frac{i}{(p / 2+k)^{2}-m^{2}+i \epsilon} \frac{i}{(p / 2-k)^{2}-m^{2}+i \epsilon} \int \frac{d^{4} k^{\prime}}{(2 \pi)^{4}} \frac{i(-i g)^{2}}{\left(k-k^{\prime}\right)^{2}-\mu^{2}+i \epsilon} \varphi\left(k^{\prime}, p\right),
$$

where $\mu$ is the exchanged-scalar mass. The BS amplitude written in terms of the NIR reads as

$$
\varphi(k, p)=-i \int_{0}^{\infty} d \gamma^{\prime} \int_{-1}^{1} d z^{\prime} \frac{g\left(\gamma^{\prime}, z^{\prime}\right)}{\left[\gamma^{\prime}+\kappa^{2}-k^{2}-(p \cdot k) z^{\prime}-i \epsilon\right]^{3}},
$$

where $\kappa^{2}=m^{2}-M^{2} / 4>0$ for bound states, with $M$ being the total energy (bound-state mass), which is usually related to the binding energy $B$, as $B=2 m-M$. Therefore, $B \in[0,2 m]$. By introducing 
the BS amplitude in terms of the NIR, (2) into (1), and performing the LF projection, we obtain the following integral equation for $g\left(\gamma^{\prime}, z^{\prime}\right)$ :

$$
\int_{0}^{\infty} d \gamma^{\prime} \frac{g\left(\gamma^{\prime}, z\right)}{\left[\gamma^{\prime}+\gamma+z^{2} m^{2}+\left(1-z^{2}\right) \kappa^{2}\right]}=\int_{0}^{\infty} d \gamma^{\prime} \int_{-1}^{1} d z^{\prime} V\left(\gamma, z ; \gamma^{\prime}, z^{\prime}\right) g\left(\gamma^{\prime}, z^{\prime}\right),
$$

where the expression for $V\left(\gamma, z ; \gamma^{\prime}, z^{\prime}\right)$, with a detailed derivation using the ladder approximation, can be found in [7]. In order to solve (3), two basis expansions were introduced for the Nakanishi weightfunction [7], in terms of Laguerre ( $\gamma$-integrations) and Gegenbauer ( $z$-integrations) polynomials. The success of this basis expansion when studying the ground state led us to consider the same polynomial basis in our numerical approach to study the excited states. In our numerical study, we found that the basis numbers for the $\gamma$ and $z$ integrations, when considering the excited states with good accuracy $\left(N_{\gamma}=26, N_{z}=10\right)$, are not too much larger than the corresponding numbers for the ground-state $\left(N_{\gamma}=14, N_{z}=10\right)$. In order to obtain the spectrum of the excited states in the numerical approach, one can slightly change the integral equation used for the ground state calculation in the following form

$$
\lambda g=\left(\frac{1}{\alpha_{g r}}\right)^{-1} \mathcal{B}^{-1}(M) \mathcal{A}(M) g,
$$

where $\mathcal{B}(M)$ and $\mathcal{A}(M)$ represent symbolically the operators in the left- and right-hand side of (3), respectively, where $\alpha_{g r}=g^{2} /\left(16 \pi m^{2}\right)$ (positive defined) is obtained from the ground-state calculation. The (4) has a pivotal role in solving the BS equation for bound states: (i) First, it allows to obtain the coupling constant for the ground state, $\alpha_{g r}$; (ii) next, by fixing $\alpha_{g r}$, one can reach the corresponding excited-state values in the spectrum, by looking for values $M=2 m-B_{g r}>M_{g r}$ such that $\lambda=1$.

By following the approach as explained above in our numerical investigation, we summarize our results for the behavior of the coupling constant as function of the binding energy in figure 1 . In this figure, we present three panels corresponding to three different values of the mass of the exchanged boson $(\mu=0.01 \mathrm{~m}, 0.1 \mathrm{~m}$ and $0.5 \mathrm{~m})$. In each panel our results are given for the ground and first two excited states. Once given $\mu$, in order to identify the possible excited states, one should first obtain the coupling for the ground state and next look for the corresponding excited states. Therefore, the relevant parts of the panels are constrained by the corresponding maximum value of the groundstate energy $(B \leq 2 m)$. As in the non-relativistic case with Yukava potential, the number of excited states is finite for $\mu \neq 0$, increasing only when $\mu \ll m$ is approaching zero [17]. Theoretically, an infinite number of states is expected for $\mu=0$ (Coulomb limit). However, as we can verify in the next section, this number increases slowly as $\mu$ is being reduced, with the corresponding coupling $\alpha_{g r}$ being adjusted by the ground-state. As shown in figure 1, the value of $\alpha_{g r}$ is restricted to a maximum value defined by $B \leq 2 \mathrm{~m}$, such that in case that $\mu=0.5 \mathrm{~m}$ only one excited state can be found.

\section{Comparison between the Minkowski and Euclidean eigenvalues}

Here we provide the results we have obtained for a comparison between the eigenvalues in the Minkowski space, using the NIR, with those obtained by solving the Euclidean BS equation. With $B(n)(n=0,1,2,3 .$.$) defined as the binding energy of the ground and excited states, where B(0)=B_{g r}$, we choose $B(0)=1.9 \mathrm{~m}$ for the binding energy of the ground state, using different mass values $\mu$ for the exchanged boson. This choice of a strongly-bound ground-state energy is motivated by the fact that the spectrum will be more sensitive to relativistic effects. Our main results are displayed in table 1 where we can observe a very satisfactory agreement between the values obtained in both Minkowski and Euclidean spaces, which provides a further reliability of the method. As an expected feature of 

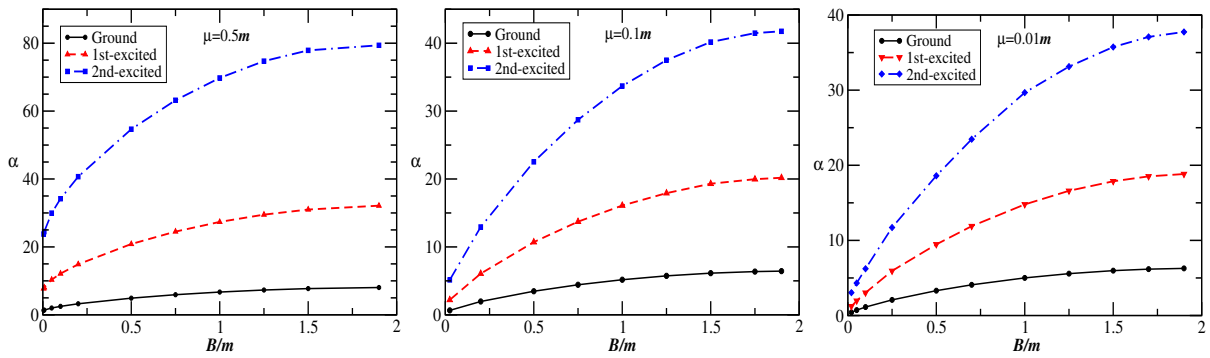

Figure 1. Behavior of the coupling constant $\alpha$ as function of the binding energy $B$, in units of $m$, for three different values of the mass $\mu$ of the exchanged boson, considering the ground and first two excited states.

the relativistic spectrum one can observe that, as $\mu$ increases the number of excited states decreases. On the other side, when $\mu$ is reduced approaching to zero, we can observe an increasing number of excited states (toward infinite for $\mu=0$ ), in agreement with the non-relativistic spectrum [17].

Table 1. Comparison of the spectra obtained in the Euclidean and Minkowski spaces [14], for the binding energies $B(n)$ of two boson with mass $m$, by varying the mass $\mu$ of the exchanged boson and, consequently, the value of the coupling $\alpha_{g r}$. In this case, the value of the ground-state binding energy was fixed to $B(0)=1.9 \mathrm{~m}$.

\begin{tabular}{cccc}
\hline \hline$\left(\mu / m, \alpha_{g r}\right)$ & & Minkowski & Euclidean \\
\hline \hline \multirow{2}{*}{$(0.05,6.324)$} & $B(1) / m$ & 0.259 & 0.258 \\
& $B(2) / m$ & 0.090 & 0.090 \\
\hline \multirow{2}{*}{$(0.1,6.437)$} & $B(1) / m$ & 0.221 & 0.220 \\
& $B(2) / m$ & 0.050 & 0.051 \\
\hline$(0.5,8.047)$ & $B(1) / m$ & 0.0082 & 0.0082 \\
\hline \hline
\end{tabular}

The energy ratios $B(n) / B(0)$ are also displayed in figure 2, showing the corresponding behavior as we vary the mass values of the exchanged boson $\mu$. We consider the first two excited states, with $B(0)=0.3 m$ (left panel) and $0.5 m$ (right panel). The non-relativistic limits are also being presented for $\mu \rightarrow 0$ in order to verify the consistency of the relativistic results. For that, we consider the analytic expression obtained in the Euclidean space for the bound state of two spinless bosons exchanging a massless scalar boson. In lower orders of $\alpha$, this expression is given by [18]

$$
B(n)=\frac{m}{4} \frac{\alpha^{2}}{(n+1)^{2}}\left[1+\frac{4 \alpha}{\pi} \ln \alpha\right]+\ldots \quad(n \geq 0) .
$$

The first term corresponds to the non-relativistic limit and it is reproduced by the energy ratios displayed in figure 2 for the first- and second-excited states in the limit $\mu=0$. Notice that the logarithmic corrections are canceled when computing the energy ratios. Moreover, the agreement of the NIR results with this expansion given by (5) can also be verified when considering smaller values of $B(0)$, as observed from table 2 . In this table, we display a set of binding energies in order to verify the range the validity of the expansion (5). One should notice that the agreement of the NIR results with the analytical expression is better for $B(0) \leq 0.02 \mathrm{~m}$, which is expected, since (5) was obtained by performing a standard perturbative expansion in $\alpha$. 

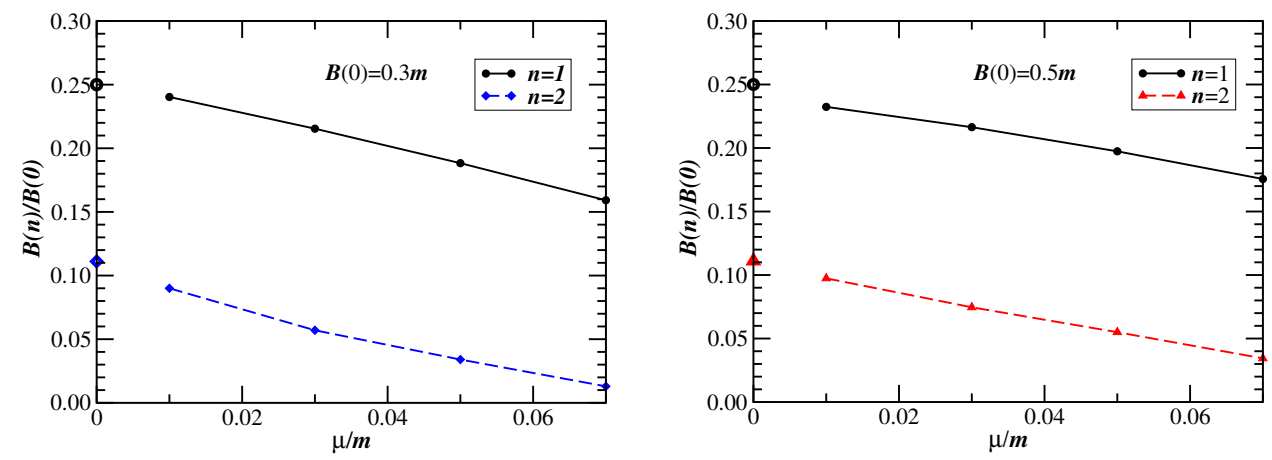

Figure 2. Energy ratios $B(n) / B(0)$ (where $B(0)$ is the ground-state energy and $n=1,2$ ) are shown for $\mu / m$. As indicated in the panels, we have fixed $B(0)$ to $0.3 \mathrm{~m}$ (left panel) and $0.5 \mathrm{~m}$ (right panel), with the first excited state given with solid lines and second excited state with dashed lines. The symbols on the lines indicate the positions where we have the values obtained through (4). At the origin, as indicated inside both panels, we can reach the corresponding non-relativistic limits expressed by (5) for the ratio $B_{n r}(n) / B_{n r}(0)=1 /(n+1)^{2}$ : $B_{n r}(1) / B_{n r}(0)=0.25$ and $B_{n r}(2) / B_{n r}(0)=0.11$. (Right panel extracted from [14]).

The results in the non-relativistic limit, given by the first term of (5) and displayed in the fourth column of table 2, show the obvious limitation of (5) to small values of $\alpha$ as compared with the third column, and considerable deviations from the results obtained with the NIR. Also, the relativistic results for the energy levels are consistently smaller than the corresponding non-relativistic ones, not only for small values of $\alpha$ but also for larger couplings, as in case of the extrapolated results shown in figure 2 for the ratios (where the $\alpha_{g r}$ 's are larger than one). As visually noticed, the extrapolated result is better verified in case that $B(0)=0.3 \mathrm{~m}$ than in the case that $B(0)=0.5 \mathrm{~m}$. The quite good results obtained for the extrapolated values of the energy ratios indicate that the expected deviations for the energies, verified in (5), are mainly canceled for the ratios in the limit $\mu=0$. As in this limit the relativistic ratios between the excited bound-state energies of the spectrum $B(n) / B(0)$ are not larger than the corresponding non-relativistic ratios, being practically the same $B(n) / B(n-1)=(1+1 / n)^{2}$, an infinite number of bound-state energies should also be verified for the relativistic case when $\mu=0$, such that $\lim _{n \rightarrow \infty} B(n)=B(n-1)$, with a finite number given in case the exchange boson has $\mu \neq 0$.

\section{Momentum space valence wave function}

Once the Nakanishi weight-function is obtained by solving (3), we can obtain the corresponding LF wave function. In terms of the variables $\xi$ and $\mathbf{k}_{\perp}$, where $\xi \equiv(1-z) / 2$ and $k_{\perp} \equiv \sqrt{\gamma}$, the LF wave function reads,

$$
\psi\left(\xi, \mathbf{k}_{\perp}\right)=\frac{1}{\sqrt{2}} \xi(1-\xi) \int_{0}^{\infty} d \gamma^{\prime} \frac{g\left(\gamma^{\prime}, 1-2 \xi\right)}{\left[\gamma^{\prime}+k_{\perp}^{2}+\kappa^{2}+(1-2 \xi)^{2} \frac{M^{2}}{4}\right]^{2}} .
$$

In figure 3, we show the behavior of this LF wave function for the ground (two upper panels) and first excited (two lower panels) states. The ground-state wave function is given for $B(0)=1.9 \mathrm{~m}$, with $\mu=0.1 \mathrm{~m}$ and $\alpha_{g r}=6.437$, with the corresponding first-excited state emerging at $B(1)=0.22 \mathrm{~m}$. The wave-function behaviors are presented in terms of the variable $k_{\perp}$ for some given values of $\xi$ (two 
Table 2. By considering the limit $\mu=0$, the coupling $\alpha_{g r}$ (1st column) with the corresponding ground-state energies are shown by the results obtained from (i) the NIR approach (2nd column); (ii) the expansion (5) (3rd column); (iii) and the corresponding non-relativistic limits (4th column), obtained from the leading term of (5).

\begin{tabular}{cccc}
\hline \hline$\alpha_{g r}$ & $B(0) / m[\mathrm{NIR}]$ & $B(0) / m[(5)]$ & $B_{n r}(0) / m$ \\
\hline \hline 0.272 & 0.010 & 0.010 & 0.018 \\
0.344 & 0.015 & 0.016 & 0.030 \\
0.401 & 0.020 & 0.021 & 0.040 \\
0.460 & 0.025 & 0.029 & 0.053 \\
0.519 & 0.030 & 0.038 & 0.067 \\
0.621 & 0.040 & 0.060 & 0.096 \\
0.711 & 0.050 & 0.087 & 0.126 \\
\hline \hline
\end{tabular}
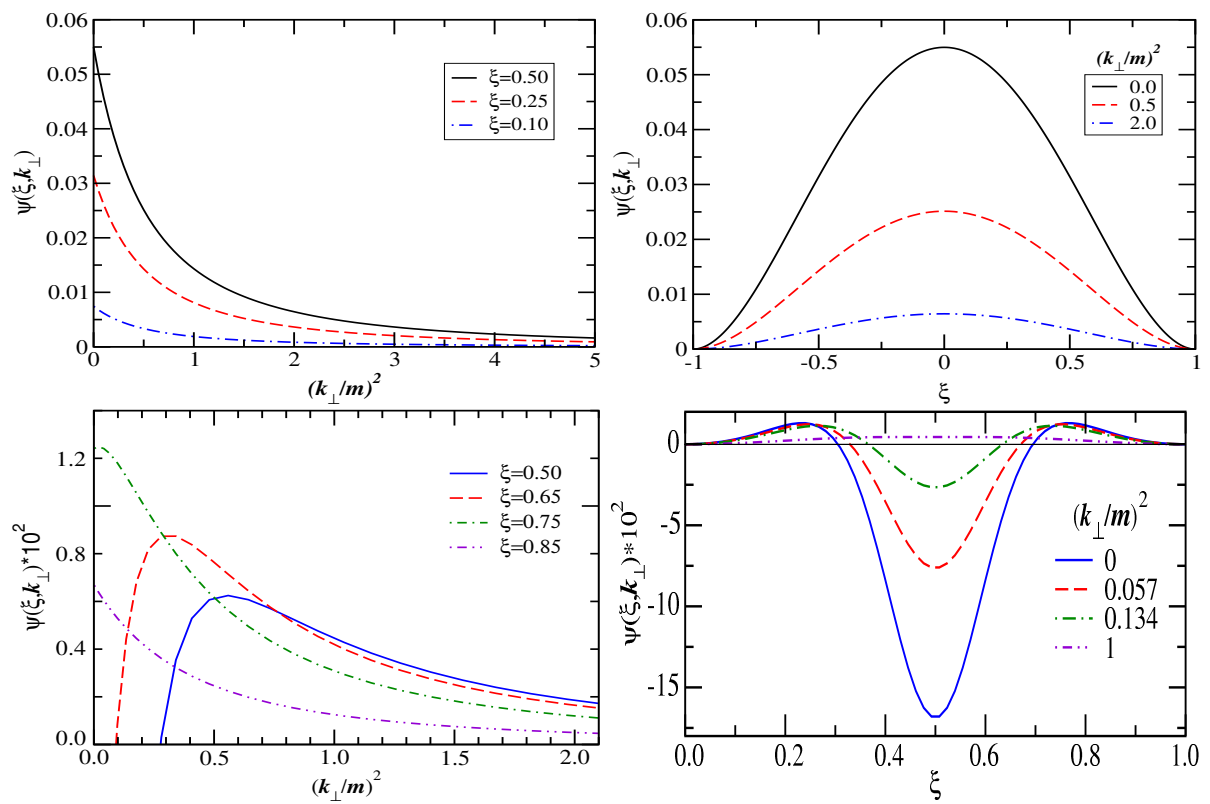

Figure 3. $\psi\left(\xi, \mathbf{k}_{\perp}\right)$ as function of $\mathbf{k}_{\perp}$ for fixed values of $\xi$ (left panels) and as function of $\xi$ for fixed values of $\left(\mathbf{k}_{\perp} / m\right)^{2}$ (right panels). For $\mu=0.1 \mathrm{~m}$, the plots are given for the ground state with $B(0)=1.9 m$ (upper frames) and for the first-excited state, $B(1)=0.22 \mathrm{~m}$ (lower frames). (For more details, see [14]).

left panels) and in terms of the variable $\xi$ for some fixed values of $k_{\perp}$ (two right panels). $>$ From the plotted results for the excited state (lower panels of figure 3), one can verify the expected node structure typical for an excited state. In addition, for the second-excited state, two nodes can be verified in the corresponding wave function (not shown here, but given in [14]).

By an inspection of the lower panels of figure 3, for the first-excited state the node structure is present for $\left(k_{\perp} / m\right)^{2}<1$ and $\xi<0.75$, being symmetric with respect to $\xi=1 / 2$. In particular, the node structure moves toward $\xi=1 / 2$ as $k_{\perp}$ increases. Such behavior can be naively expected by the 
relation between the Cartesian variables for three-momenta with the corresponding LF ones:

$$
M^{2}=\left[\sqrt{\mathbf{p}_{1}^{2}+m^{2}}+\sqrt{\mathbf{p}_{1}^{2}+m^{2}}\right]^{2}-\left(\mathbf{p}_{1}+\mathbf{p}_{2}\right)^{2}=p^{+}\left[\frac{m^{2}+\mathbf{p}_{1}^{\perp^{2}}}{p_{1}^{+}}+\frac{m^{2}+\mathbf{p}_{2}^{\perp^{2}}}{p_{2}^{+}}\right]-\mathbf{p}_{\perp}^{2} .
$$

In the rest frame, $\mathbf{p}_{1}+\mathbf{p}_{2}=0$, and by introducing the relative coordinates we obtain

$$
\mathbf{k}^{2}=\frac{k_{\perp}^{2}+m^{2}}{4 \xi(1-\xi)}-m^{2}
$$

The first excited-state wave function has to display a node at a given fixed value of $\mathbf{k}^{2}$. Therefore, as shown by the lower panels of figure 3 , by increasing the value of $k_{\perp}$, the corresponding variable $\xi$ becomes constrained to approach $1 / 2$ (i.e. the maximal value of $\xi(1-\xi)$ ).

\section{Valence wave function in the impact-parameter space}

A convenient and useful approach to study the valence amplitude is by considering the impact parameter (IP) space [16], in connection with the Generalized Parton Distributions. This is performed by keeping the longitudinal momentum and applying the Fourier transformation over the transverse momentum of the LF wave function. Such mixed representation shows the correlation between the momentum fraction carried by the constituent particle and the corresponding probability distribution in the transverse plane. This can be particularly interesting for the analysis of fermionic systems carrying both angular momentum and spin, with a distribution correlated to the longitudinal momentum, as a reflection of the internal dynamics.

The wave function in the IP-space can be used to compute the elastic electromagnetic form factor, generalizing the well-known relation of the non-relativistic form factor to a relativistic framework. In this case, the form factor is computed as the Fourier transform of the transverse charge density, as thoroughly discussed by Miller in [19]. For a composite bosonic state, the form factor can be written as

$$
\mathcal{F}\left(Q^{2}=-q^{2}\right)=\int d^{2} \mathbf{b} \rho(\mathbf{b}) e^{-i \mathbf{b} \cdot \mathbf{q}_{\perp}},
$$

by considering the following points: (i) the momentum transfer $q^{\mu}$ is evaluated in the Breit frame with $q^{+}=0$, (ii) $Q^{2}=\mathbf{q}_{\perp}^{2}$, (iii) $\mathbf{b}$ belongs to the transverse plane or IP-space, and (iv) $\rho(\mathbf{b})$ is the impact parameter density, which is a sum of contributions from all Fock components of the LF wave function,

$$
\rho(\mathbf{b})=\rho_{\text {val }}(\mathbf{b})+\text { higher Fock states densities } \cdots .
$$

The valence term is defined through the valence wave function in the IP-space, $\phi(\xi, \mathbf{b})$, as follows

$$
\rho_{\mathrm{val}}(\mathbf{b})=\frac{1}{4 \pi} \int_{0}^{1} \frac{d \xi}{\xi(1-\xi)^{3}}|\phi(\xi, \mathbf{b} /(1-\xi))|^{2} .
$$

In (11), the IP-space valence wave function is the $2 \mathrm{D}$ Fourier transform of $\psi\left(\xi, \mathbf{k}_{\perp}\right)$, given by

$$
\phi(\xi, \mathbf{b})=\int \frac{d^{2} \mathbf{k}_{\perp}}{(2 \pi)^{2}} \psi\left(\xi, \mathbf{k}_{\perp}\right) \mathrm{e}^{\mathrm{i} \mathbf{k}_{\perp} \cdot \mathbf{b}}
$$

where $\phi(\xi, \mathbf{b})$ results to be symmetric with respect to $1-2 \xi$, as a consequence of the symmetry of $g(\gamma, z)$ under the transformation $z \rightarrow-z$. By performing the 2D Fourier transformation, the IP-space valence wave function can be written within the NIR for the s-wave state as

$$
\phi(\xi, \mathbf{b})=\frac{\xi(1-\xi)}{4 \pi \sqrt{2}} F(\xi, b),
$$


where

$$
F(\xi, b)=b \int_{0}^{\infty} d \gamma g(\gamma, 1-2 \xi) \frac{K_{1}\left(b \sqrt{\gamma+\kappa^{2}+(1 / 2-\xi)^{2} M^{2}}\right)}{\sqrt{\gamma+\kappa^{2}+(1 / 2-\xi)^{2} M^{2}}} .
$$

Therefore, the structure of the excited state was further investigated in our approach in the IP-space, with the corresponding results being presented in two panels given in figure 4 . In the left panel, we show the function $F(\xi, b)$ in terms of the $\xi$ variable, for fixed values of $m b$; and, in the right panel, in terms of $m b$, for fixed values of $\xi$. We consider the case that the binding energy is $B(1)=0.22 m$, obtained for $\mu=0.1 \mathrm{~m}$ and $\alpha_{g r}=6.437$. The moving position of the node in the $(\xi, b)$ plane, can be appreciated in both panels of the figure. As a general remark, the position of the node increases with $m b$ for $\xi$ approaching the end-points and tends to be suppressed close to such boundaries. This is the counterpart of the previous observation done for the results shown in momentum space. In addition, as one can observe in the right panel of the figure 4, there is a fast fall-off of the wave function for large values of $m b$, which indeed can be verified to have an exponential behavior, as detailed below.
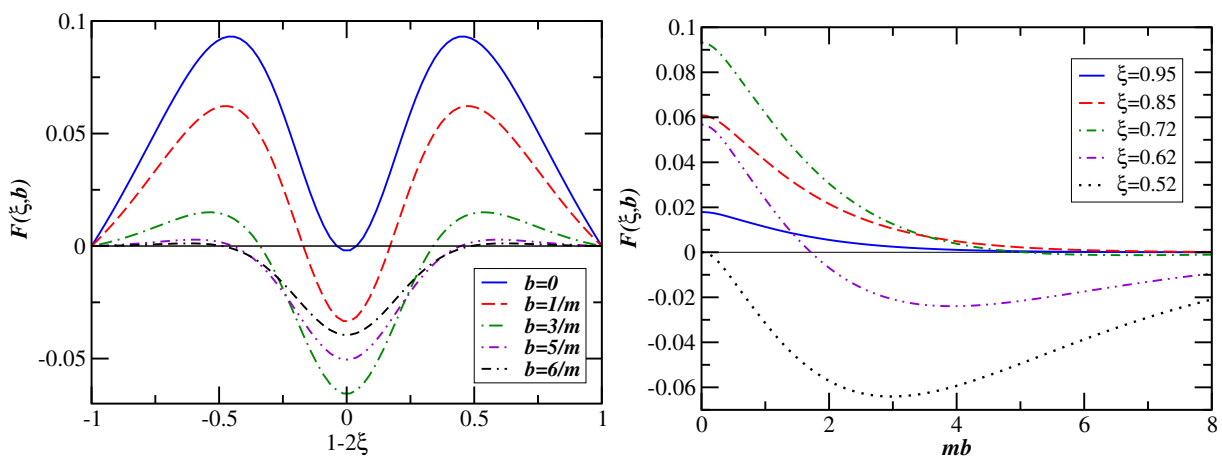

Figure 4. The first excited-state function $F(\xi, b)$, as given by (14), for $B(1)=0.22 \mathrm{~m}$, with $\mu=0.1 \mathrm{~m}$ and $\alpha_{g r}=6.437$, is shown as function of $\xi$, for some given values of $m b$ (left panel); and, as function of $m b$, for given fixed values of $\xi$ (right panel). In view of the symmetry, we should observe that all the plots in the right panel for fixed $\xi$ are exactly the same for $1-\xi$. As evidenced in both the panels, the existence of zeros for $F(\xi, b)$ occurs only for values of $m b$ not larger than $\sim 7$.

The exponentially drops out behavior in $F(\xi, b)$ can be understood by a close analysis of (14). First, from a physically-motivated requirement [19], one should note that $\phi(\xi, b)$ is finite for $b \rightarrow 0$ (see also [7]), such that

$$
\phi(\xi, b=0)=\frac{\xi(1-\xi)}{4 \pi \sqrt{2}} \int_{0}^{\infty} d \gamma \frac{g(\gamma, 1-2 \xi)}{\gamma+\kappa^{2}+(1 / 2-\xi)^{2} M^{2}}<\infty,
$$

following that one can deduce that $g(\gamma, 1-2 \xi)$ must vanish for $\gamma \rightarrow \infty$. Therefore, the relevant interval of $\gamma$ in the integral given in (14) can be taken as effectively finite. Exploring such an observation, one can extract the driving exponential fall-off of $F(\xi, b)$ in the asymptotic limit $b \rightarrow \infty$. In this limit,

$$
\left.K_{1}(x)\right|_{x \rightarrow \infty} \rightarrow\left(\frac{\pi}{2 x}\right)^{\frac{1}{2}} e^{-x}
$$

By considering the limit $b \rightarrow \infty$, we notice that the leading exponential behavior in the integral (14) is coming from values of $e^{-b \sqrt{\gamma+\kappa^{2}+(\xi-1 / 2)^{2} M^{2}}}$ with $\gamma$ close to 0 . Therefore, in such limit, we can single 
out the exponential fall-off behavior as

$$
\left.F(\xi, b)\right|_{b \rightarrow \infty} \rightarrow e^{-b \sqrt{\kappa^{2}+(\xi-1 / 2)^{2} M^{2}}} f(\xi, b),
$$

where the reduced function $f(\xi, b)$ is expected to decrease more smoothly for large values of $b$. The feature shown in (17) is typical for the wave function in coordinate representation, i.e., exponential fall-off multiplied by a polynomial function.

\section{Conclusions}

In the present contribution, we are reporting some recent investigations on the spectrum of excited states of the Bethe-Salpeter equation in $3+1$ space-time dimensions, considering the $s$-wave state in the ladder approximation, using the Nakanishi integral representation. We have found a finite number of excited states for non-zero exchanged-scalar mass, and we have successfully compared our results with the corresponding ones obtained in the Euclidean space, where obviously the Nakanishi integral representation is not assumed. Here we present some sample results obtained for the first two excited states; however the method has allowed us to obtain up to three excited states $B(n)(n=1,2 \ldots)$ for large enough ground-state binding energy $B(0)$ and small mass $\mu$ of the exchanged boson [13]. In order to verify the non-relativistic limit when the mass of the exchanged boson is approaching to zero, the ratios $B(n) / B(0)$ obtained through the Bethe-Salpeter approach are in agreement with those of the well known non-relativistic calculation. To compute these ratios for $\mu$ close to zero, an extrapolation was performed, due to numerical instabilities of the integral equation that occur for very small $B(0)$ and small mass of the exchanged boson. From our analysis of the valence wave function, which is more detailed in $[13,14]$, we have presented results for the expected node structure of the firstexcited state when plotted as function of the LF momentum variables. The structure of the impactparameter space valence wave function has also been explored, with our results presented for the firstexcited state. In particular, for large transverse distances an exponential fall-off behavior was singled out in agreement with the non-relativistic case in the 3D Euclidean space. The success in applying the NIR to the BS formalism for the spectrum of bosonic systems, as outlined here and detailed in $[13,14]$, provides enough motivation for possible extensions, as to consider these studies beyond the ladder approximation [9]. Following previous investigations on two-fermion systems within the NIR $[11,12]$, it is quite relevant also to look for the corresponding excited bound-state spectrum.

\section{Acknowledgments}

The authors thank Profs. Tobias Frederico, Vladimir Karmanov, and Giovanni Salmè for clarifying discussions along the development of works related to this communication. We also acknowledge the partial financial support received from the Brazilian funding agencies CAPES, CNPq and FAPESP.

\section{References}

[1] E.E. Salpeter, H.A. Bethe, Phys. Rev. 84, 1232 (1951)

[2] N. Nakanishi, Phys. Rev. 130, 1230 (1963). Graph Theory and Feynman Integrals (Gordon and Breach, New York, 1971)

[3] K. Kusaka and A.G. Williams, Phys. Rev. D 51, 7026 (1995)

[4] K. Kusaka, K. Simpson and A.G. Williams, Phys. Rev. D 56, 5071 (1997)

[5] V. A. Karmanov and J. Carbonell, Eur. Phys. J. A 27, 1 (2006) 
[6] T. Frederico, G. Salmè and M. Viviani, Phys. Rev. D 85, 036009 (2012)

[7] T. Frederico, G. Salmè and M. Viviani, Phy. Rev. D 89, 016010 (2014)

[8] J. Carbonell and V. A. Karmanov, Eur. Phys. J. A 27, 11 (2006)

[9] V. Gigante, J.H.A. Nogueira, E. Ydrefors, C. Gutierrez, V.A. Karmanov and T. Frederico, arXiv:1611.03773 (2016)

[10] V. Sauli and J. Adam, Phys. Rev. D 67, 085007 (2003)

[11] J. Carbonell, V. A. Karmanov, Eur. Phys. J. A 46, 387 (2010)

[12] W. de Paula, T. Frederico, G. Salmè and M. Viviani, Phys. Rev. D 94, 071901 (2016)

[13] C. Gutierrez, Minkowski space Bethe-Salpeter equation within Nakanishi representation, $\mathrm{PhD}$ thesis IFT-T.007/16 (Instituto de Física Teórica, Universidade Estadual Paulista, 2016)

[14] C. Gutierrez, V. Gigante, T. Frederico, G. Salmè, M. Viviani, L. Tomio, Phys. Lett. B 759, 131 (2016)

[15] M. Burkardt, Int. J. Mod. Phys. A 18, 173 (2003)

[16] M. Diehl, Phys. Rep. 388, 41 (2003)

[17] E. R. Vrscay, Phys. Rev. A 33, 1433 (1986)

[18] G. Feldman, T. Fulton and J. Townsend, Phys. Rev. D 7, 1814 (1973)

[19] G. A. Miller, Annu. Rev. Nucl. Part. Sci. 60, 25 (2010) 